\title{
Crossover from coherent to incoherent electronic excitations in the normal state of $\mathrm{Bi}_{2} \mathrm{Sr}_{2} \mathrm{CaCu}_{2} \mathrm{O}_{8+\delta}$
}

\author{
A. Kaminski, ${ }^{1,2}$ S. Rosenkranz, ${ }^{1,2}$ H. M. Fretwell, ${ }^{3}$ Z. Li, ${ }^{4}$ H. \\ Raffy, ${ }^{4}$ M. Randeria, ${ }^{5}$ M. R. Norman, ${ }^{2}$ and J. C. Campuzano ${ }^{1,2}$ \\ ${ }^{1}$ Department of Physics, University of Illinois at Chicago, Chicago, Illinois 60607, USA \\ ${ }^{2}$ Materials Science Division, Argonne National Laboratory, Argonne, Illinois 60439, USA \\ ${ }^{3}$ Department of Physics, University of Wales Swansea, Swansea, SA2 8PP, UK \\ ${ }^{4}$ Laboratoire de Physique des Solides, Universit Paris-Sud,91405 Orsay, France \\ ${ }^{5}$ Tata Institute for Fundamental Research, Homi Bhabha Road, Mumbai 400005, India
}

(Dated: November 1, 2018)

\begin{abstract}
Angle resolved photoemission spectroscopy (ARPES) and resistivity measurements are used to explore the overdoped region of the high temperature superconductor $\mathrm{Bi}_{2} \mathrm{Sr}_{2} \mathrm{CaCu}_{2} \mathrm{O}_{8+\delta}$. We find evidence for a new crossover line in the phase diagram between a coherent metal phase for lower temperatures and higher doping, and an incoherent metal phase for higher temperatures and lower doping. The former is characterized by two well-defined spectral peaks in ARPES due to coherent bilayer splitting and superlinear behavior in the resistivity, whereas the latter is characterized by a single broad spectral feature in ARPES and a linear temperature dependence of the resistivity.

PACS numbers: 74.25.Dw, 74.25.Fy, 74.72.Hs, 79.60.Bm
\end{abstract}

The normal state of optimal and underdoped high temperature superconductors (HTSCs) exhibits anomalous transport and spectroscopic properties which have long been recognized as one of the central mysteries of the field [1, 2]. The electronic excitations are unlike those of conventional metals, where one can think of dressed electrons - quasiparticles. Instead, the response in the normal state of the HTSCs is incoherent, with no identifiable single particle like excitations. The key question is how the strange metal evolves into the conventional one at high doping. Some models propose that the incoherent normal metal represents a new state of matter [3], with a crossover to more conventional behavior 顿. Others suggest that the incoherent state is a result of underlying competing interactions, and therefore its behavior evolves continuously from the conventional one [5].

Here, using photoemission and resistivity, we study the issue of the coherence of the electronic excitations. We find a new crossover line in the phase diagram of the HTSCs between the low temperature, overdoped side with coherent electronic excitations, and the high temperature, underdoped side, where this coherence is lost, and therefore conclude that indeed the two states are qualitatively different.

For our measurements, we have chosen $\mathrm{Bi}_{2} \mathrm{Sr}_{2} \mathrm{CaCu}_{2} \mathrm{O}_{8+\delta}$ (Bi2212), which has the advantage that it can be prepared over a wide range of doping, and is ideal for angle resolved photoemission (ARPES) given its quasi two dimensionality. Additionally, it possesses two $\mathrm{CuO}_{2}$ layers (a bilayer), and thus the issue of coherence can be probed by not only examining its planar properties as a function of doping and temperature, but also by looking for the presence of bilayer splitting; if the motion of electrons within the bilayer is coherent, then we expect the formation of antibonding (A) and bonding (B) states. These are the antisymmetric and symmetric combinations of the layer wavefunctions with energies $\epsilon_{A(B)}(\mathbf{k})=\epsilon(\mathbf{k}) \pm t_{\perp}(\mathbf{k})$ where $\epsilon(\mathbf{k})$ is the planar dispersion and $t_{\perp}(\mathbf{k})$ the interplanar coupling. As the $\mathrm{CuO}_{2}$ planes in Bi2212 are separated by only $3.17 \AA$, electronic structure calculations predict a sizable bilayer splitting of order $0.3 \mathrm{eV}$ [6]. In the coherent regime, the primary effect of interactions would be to renormalize the splitting to a smaller value, without qualitatively affecting the spectrum. On the other hand, if the interactions are sufficiently strong, then we expect the coherent behaviour within each plane, as well as the coherent motion within the bilayer, to be destroyed 11. The in-plane effect would be reflected in both the ARPES spectral lineshape and in the temperature dependence of the planar transport. The out-of-plane effect would be a loss of the coherent bilayer splitting.

The ARPES and resistivity data were obtained on high quality epitaxially grown thin films of Bi2212 [7]. These films have the very useful feature of displaying small signals from the structural superlattice distortion $(<3 \%)$, which otherwise would complicate [8] the interpretation of ARPES data in the vicinity of the $(\pi, 0)$ point of the Brillouin zone. This is particularly important for us, since bilayer splitting is maximal at $(\pi, 0)$ [9, 10, 11, 12, as shown in panel a of Fig. 1.

ARPES measurements were carried out at the Synchrotron Radiation Center in Wisconsin with an energy resolution of $30 \mathrm{meV}$ and a momentum resolution of 0.01 Åusing our SES50 analyzer and undulator 4 meter NIM beamline. The ARPES intensity as a function of the planar momentum $\mathbf{k}$ and energy $\omega$ (measured with respect to the chemical potential) is given by [13] $I(\mathbf{k}, \omega)=I_{0}(\mathbf{k}) f(\omega) A(\mathbf{k}, \omega)$ (convolved with the resolution function). Here, $I_{0}$ is an intensity prefactor, 


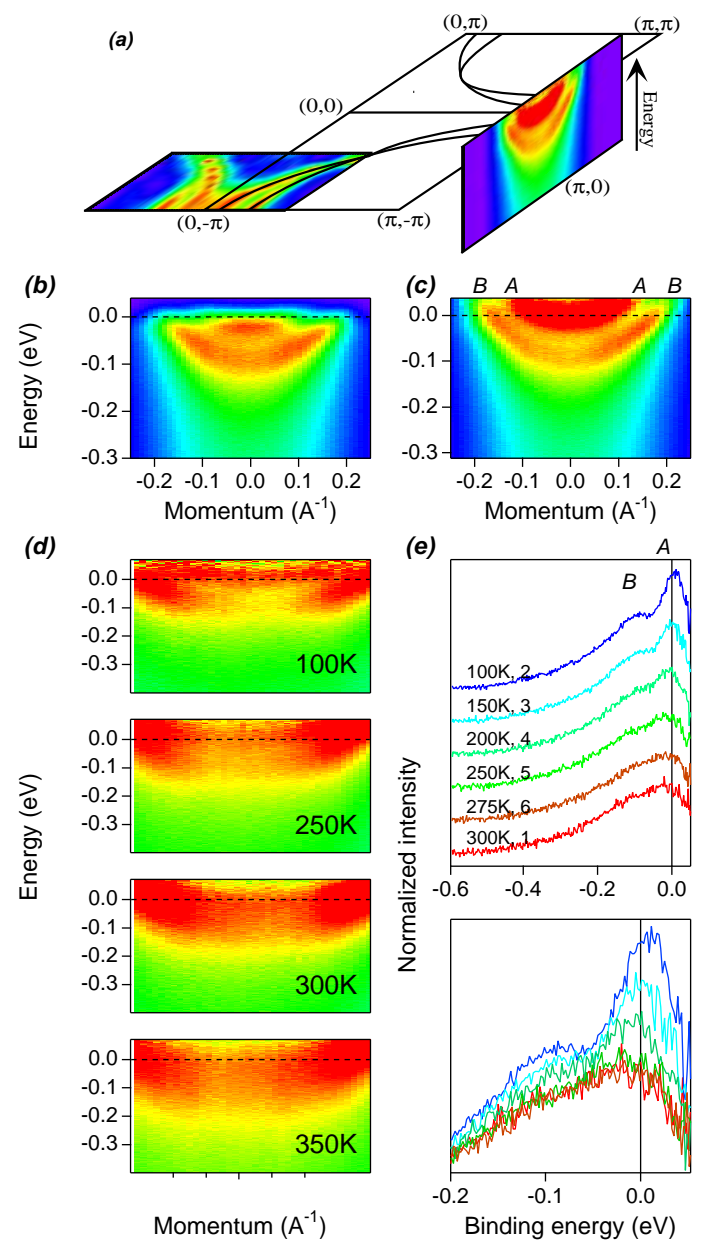

FIG. 1: ARPES data for overdoped $\left(T_{c}=52 \mathrm{~K}\right)$ Bi2212 samples. (a) Intensity versus momentum and energy for $\mathrm{T}=100 \mathrm{~K}$. The two solid curves represent the bilayer split Fermi surface. (b) Intensity for momenta along $(\pi, 0)-(\pi, \pi)$ at $\mathrm{T}=100 \mathrm{~K}$, with plots centred at $(\pi, 0)$. (c) The same data divided by the Fermi function. (d) Same as (c), but at various temperatures. (e) Spectrum at $(\pi, 0)$ (divided by the Fermi function) at various temperatures. All curves are overlapped on the bottom of the panel to demonstrate lack of temperature dependence of the lineshape above $250 \mathrm{~K}$.

$f$ the Fermi function, and $A$ the single particle spectral function, which measures the probability of removing or adding an electron from the system. The peak in $A(\mathbf{k}, \omega)$ measures the energy of the electronic excitation, while its linewidth is inversely proportional to the lifetime. Since the ARPES lineshape is "chopped off" by the Fermi function, we choose in some cases to divide our data by a resolution broadened Fermi function, obtained by fitting the leading edge of a polycrystalline $\mathrm{Au}$ in contact with the sample. This procedure allows us to focus directly on $A(\mathbf{k}, \omega)$ (albeit approximately because of the resolution convolution).

In panel (b) of Fig. 1 we plot raw ARPES data for an overdoped (OD) sample $\left(T_{C}=52 \mathrm{~K}\right)$ at $\mathrm{T}=100 \mathrm{~K}$, along
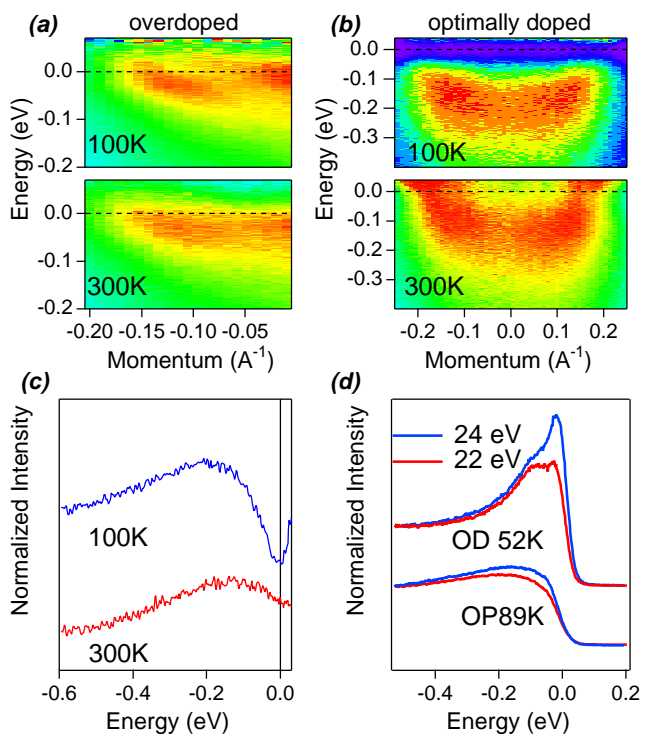

FIG. 2: ARPES data for samples at various dopings. (a) As in Fig. $1(\mathrm{~d})$, but for an overdoped $\left(T_{C}=75 \mathrm{~K}\right)$ sample. (b) As in Fig. $1(\mathrm{~d})$, but for an optimal doped $\left(T_{C}=89 \mathrm{~K}\right)$ sample (at $100 \mathrm{~K}$ the intensity at the chemical potential is suppressed due to the pseudogap). (c) Spectrum at $(\pi, 0)$ (divided by the Fermi function) for an optimal doped $\left(T_{C}=89 \mathrm{~K}\right)$ sample. (d) Raw data at $(\pi, 0)$ at two different photon energies for an overdoped ( $T_{C}=52 \mathrm{~K}$ sample) and an optimal doped $\left(T_{C}=\right.$ $89 \mathrm{~K}$ ) sample at $\mathrm{T}=100 \mathrm{~K}$.

a momentum cut centred at the $(\pi, 0)$ point of the Brillouin zone. In addition, in panel (c), we plot the same data divided by the Fermi function, which approximates the true spectral function. The data in panel (c) reveal two dispersing bands due to the bilayer splitting, with the $\mathrm{A}$ band close to, and the $\mathrm{B}$ band well below, the chemical potential. In panel (d), we show data like that in (c) (divided by the Fermi function) for another sample with the same $T_{C}$ as a function of temperature. The bilayer splitting can clearly be seen at $100 \mathrm{~K}$, however above $250 \mathrm{~K}$ the two bands are no longer observed. To obtain more precise information, in panel (e) we show the temperature dependence of the spectral function at $(\pi, 0)$ as a function of energy (raw data divided by the Fermi function). The sample was temperature-cycled when taking the data to ensure that the observed effect is intrinsic and not due to the sample aging (the numbers in the legend indicate the order of measurement). At $100 \mathrm{~K}$, one sees clearly the presence of two peaks, a sharp A peak near the chemical potential, and a broader B peak at about $100 \mathrm{meV}$ below. As the temperature is increased, the peaks broaden and lose intensity, until only a single broad peak remains at $250 \mathrm{~K}$. At the bottom of the panel (e), we plot the curves for all temperatures without an offset to show that lineshape changes occur only up to $250 \mathrm{~K}$. Based on this, we argue that above $250 \mathrm{~K}$ the system no longer exhibits 


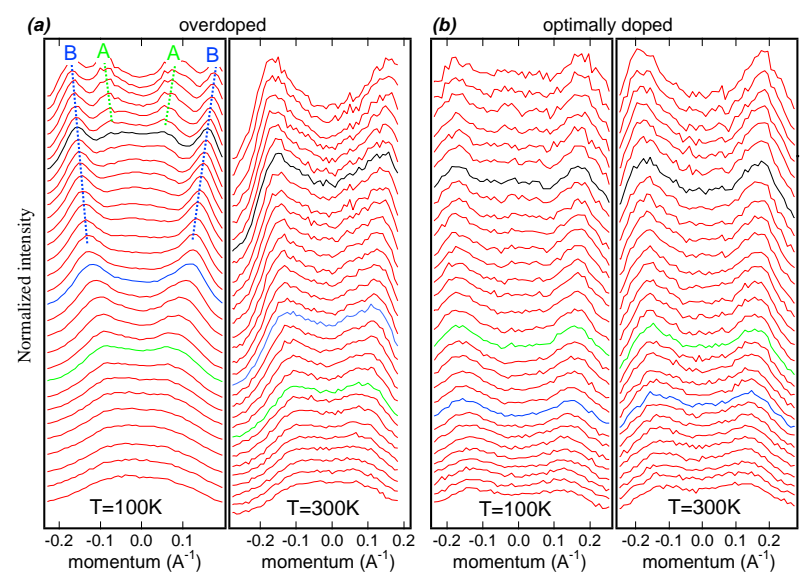

FIG. 3: Momentum distribution curves (MDCs) along $(\pi, 0)-(\pi, \pi)$ for various energies. The black curves are at the chemical potential, the blue at $-50 \mathrm{meV}$ and the green at $-100 \mathrm{meV}$. The curves for the energies above $-50 \mathrm{meV}$ are spaced every $5 \mathrm{meV}$ and remaining curves every $10 \mathrm{meV}$. (a) Overdoped $\left(T_{C}=52 \mathrm{~K}\right)$ sample at $100 \mathrm{~K}$ ("A" and green dotted lines mark antibonding peaks, "B" and blue dotted lines bonding peaks) and $300 \mathrm{~K}$. (b) Optimal $\left(T_{C}=89 \mathrm{~K}\right)$ sample at $100 \mathrm{~K}$ and $300 \mathrm{~K}$. The data are quite noisy at $100 \mathrm{~K}$ close to the chemical potential because of the intensity being suppressed due to the pseudogap.

coherent excitations, both in regards to inverse lifetime (spectral peak widths) and bilayer splitting (appearance of two separate spectral peaks). That is, the data indicate that both in-plane and out-of-plane coherence are lost.

We now examine the issue of coherence as a function of doping. In panel (a) of Fig. 2, we show data like in Fig. 1(d), but for an overdoped $\left(T_{C}=75 \mathrm{~K}\right)$ sample. Again, note the presence of bilayer splitting at $100 \mathrm{~K}$ which is not visible at $300 \mathrm{~K}$. We can contrast this behaviour with that of an optimally doped sample $\left(T_{C}=\right.$ 89K) shown in panel (b), where the intensity plots do not indicate the presence of bilayer splitting, even at $100 \mathrm{~K}$. This is further illustrated in panel (c), where again the spectrum at $(\pi, 0)$ (divided by the Fermi function) is shown. At $100 \mathrm{~K}$, only a single broad peak is seen, with no presence of bilayer splitting, indicating incoherent behaviour. Instead, a pseudogap is seen, centred at the chemical potential, which fills in as the temperature is increased. An important check can be made by analyzing the photon energy dependence of the data. It has been recently observed that the spectral lineshape changes as a function of photon energy for overdoped samples due to the relative weighting of the $A$ and $B$ peaks [11, 12]. This is clearly seen in Fig. 2(d), where data at $(\pi, 0)$ for the overdoped sample of Fig. 1 is shown for two different photon energies. In contrast, for the optimal doped sample, only a very small change with photon energy is observed (panel d), indicating the absence of bilayer splitting.
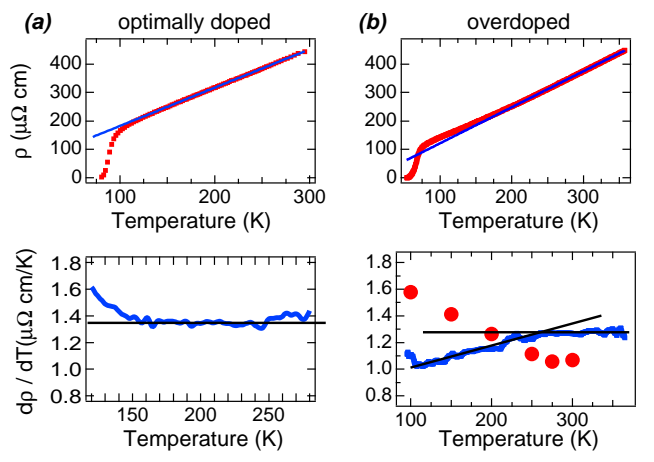

FIG. 4: Resistivity data. The blue lines denote linear $\mathrm{T}$ fits to the high temperature data for (a) optimal doped $\left(T_{C}=\right.$ $89 \mathrm{~K})$ and $(\mathrm{b})$ overdoped $\left(T_{C}=52 \mathrm{~K}\right)$ samples (top graphs). The bottom plots are the temperature derivative of the resistivity for the two dopings, with black lines guides to the eye. Red dots on bottom right plot are the ARPES intensities from Fig. 1(e).

It is also useful to plot the data as a function of momentum for fixed energy (momentum distribution curve, or MDC). In Fig. 3 we show such plots for a few values of the binding energy, at low and high temperatures, for two samples, an overdoped sample like that in Fig. 1, and another optimal doped sample $\left(T_{C}=89 \mathrm{~K}\right)$. At $100 \mathrm{~K}$ and energies close to the chemical potential, the overdoped MDC has four peaks (panel a). The two peaks closest to the centre of the plot correspond to the A band, while the two peaks on the outside correspond to the $\mathrm{B}$ band. As the binding energy increases, the two A peaks approach each other and then merge into a single peak, which then disappears at still higher energies. This is the expected behaviour of MDCs close to the bottom of a band, and is also observed for the B peak at even higher binding energies. However, at high temperatures only two peaks are observed, regardless of binding energy (panel a). This can be contrasted with the optimally doped sample, where only two peaks are visible in the MDCs, whether at $100 \mathrm{~K}$ or $300 \mathrm{~K}$ (panel b).

We now connect our ARPES observations with transport data taken on the same films. The resistivity was determined using the standard four-probe method. In Fig. 4 we plot the resistivity as a function of temperature for the optimally doped (panel a) and overdoped (panel b) samples of Fig. 3, with the black line a linear fit to the high temperature data. Both samples at high temperatures exhibit a linear $\mathrm{T}$ resistivity, which has been linked to the absence of coherent quasiparticles 11, 2. For the optimally doped sample, this behaviour continues to near $T_{C}$, with the rounding just above $T_{C}$ due to fluctuation effects. In contrast, the overdoped 


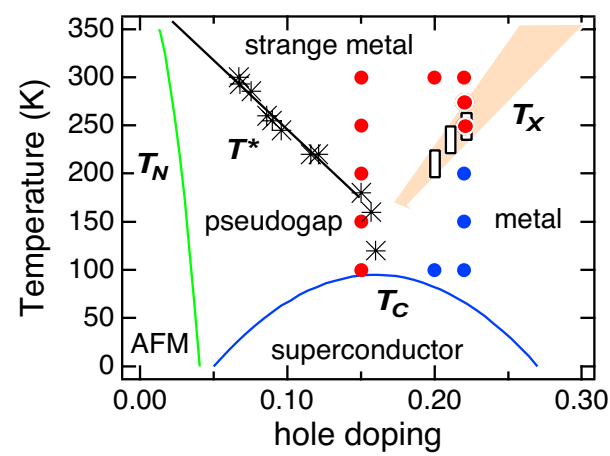

FIG. 5: Phase diagram for the HTSCs. The strange metal/pseudogap transition (black stars) and strange metal/metal crossover (black squares) are obtained from the departure from linear $\mathrm{T}$ resistivity. The open red circles correspond to ARPES data where bilayer splitting was not observed, the blue dots correspond to ARPES data showing bilayer splitting.

sample shows strong deviations from linearity, which set in at about $250 \mathrm{~K}$. These results can be understood more easily by plotting the derivative of the resistivity, which emphasizes the strength of the inelastic scattering contribution. One can see that while the optimally doped sample shows a constant derivative, the overdoped sample shows a change in derivative at $250 \mathrm{~K}$. Below this temperature, the derivative monotonically decreases. The superlinear behaviour of the resistivity below $250 \mathrm{~K}$ indicates the presence of coherent excitations. We note the strong correlation of these observations with ARPES. In the optimally doped sample, sharp spectral peaks only begin to appear at temperatures slightly above $T_{C}$. And in the overdoped sample (Fig. 1e), the sharp A peak disappears above $250 \mathrm{~K}$. As we show in the bottom right panel of Fig. 4, the ARPES intensity is inversely related to $d \rho / d T$. In particular, the intensity becomes constant when the resitivity becomes linear.

Based on our results, we show in Fig. 5 a proposed phase diagram for the HTSCs. The crossover between the pseudogap phase and the strange metal phase has been studied in the past, both by ARPES [14] and transport [7]. What we have shown here is the presence of a new crossover line, between a conventional metal phase on the overdoped side of the phase diagram and the strange metal phase, seen from both spectroscopic and transport measurements. The two crossover lines (pseudogap/strange metal and metal/strange metal) were determined by the departure from linear $\mathrm{T}$ resistivity. In all cases, the crossover lines were verified by ARPES, with the pseudogap line being determined by the closing of the leading edge gap at $(\pi, 0)$, and the metal line by the loss of the sharp (A) peak at $(\pi, 0)$.

The crossover line we have found from the loss of coherence has been long predicted on theoretical grounds. Slave boson studies of the t-J model [4, 15] predict a phase diagram very similar to Fig. 5, with the crossover line between the strange metal and metal phases marking the "condensation of holons" (i.e., for temperatures below this, the doped holes have phase coherence). However, a similar crossover to the one observed here may also be expected near a quantum critical point [16], with the "ordered" region corresponding to the pseudogap, the disordered region the conventional metal, and the quantum critical regime the strange metal phase. Further studies are needed to distinguish these possibilities.

In conclusion, our data show the presence of a coherent normal metal in overdoped samples, and that this state crosses over into an incoherent metal at higher temperatures. We emphasize that the ARPES data indicate a loss of both in-plane and out-of-plane coherence. Furthermore, this crossover temperature increases with doping. Our studies indicate that the normal state of the HTSCs, with its various phases, is a much richer field of study than even its exotic superconducting state, and has strong implications for the many body theory of electrons in reduced spatial dimensions.

This work was supported by the NSF DMR 9974401 and the U.S. DOE, Office of Science, under Contract No. W-31-109-ENG-38. The Synchrotron Radiation Center is supported by NSF DMR 9212658. SR was supported in part by the Swiss National Science Foundation, and MR by the Indian DST through the Swarnajayanti scheme. We acknowledge helpful discussions with P.D. Johnson.

[1] P.W. Anderson, The Theory of Superconductivity in the High- $T_{c}$ Cuprates (Princeton Univ. Press, Princeton, 1997).

[2] C. M. Varma, P.B. Littlewood, S. Schmitt-Rink, E. Abrahams, A.E. Ruckenstein, Phys. Rev. Lett. 63, 1996 (1989).

[3] P.W. Anderson, Science 235, 1196 (1987).

[4] H. Fukuyama, Prog. Theor. Phys. Suppl. 108, 287 (1992).

[5] R.B. Laughlin, Adv. Phys. 47, 943 (1998).

[6] S. Massidda, J.J. Yu, A.J. Freeman, Physica C 152, 251 (1988).

[7] Z. Konstantinovic, Z.Z. Li, H. Raffy, Physica B 259-261, 567 (1999).

[8] H. Ding et al., Phys. Rev. Lett. 76, 1533 (1996).

[9] O.K. Andersen, A.I. Liechtenstein, O. Jepsen, F. Paulsen, J. Phys. Chem. Solids 56, 1573 (1995).

[10] S. Chakravarty, A. Sudbo, P.W. Anderson, S. Strong, Science 261, 337 (1993).

[11] D.L. Feng et al., Phys. Rev. Lett. 86, 5550 (2001).

[12] Y.D. Chuang et al., Phys. Rev. Lett. 87, 117002 (2001).

[13] M. Randeria et al., Phys. Rev. Lett. 74, 4951 (1995).

[14] J.C. Campuzano et al., Phys. Rev. Lett. 83, 3709 (1999).

[15] P.A. Lee, N. Nagaosa, Phys. Rev. B 46, 5621 (1992).

[16] C.M. Varma, Phys. Rev. B 55, 14554 (1997). 